\title{
The Actuation Response of Model SMA Hybrid Laminates
}

C.M. Friend and N. Morgan

Cranfield University, Shrivenham Campus, Swindon, England, U.K.

\begin{abstract}
This paper presents the results from an experimental study on the actuation response of composite laminates containing Shape-Memory Alloy (SMA) actuators. Unidirectional glassfibre reinforced epoxide laminates were constructed containing Ni-Ti SMA actuators orientated parallel to the glass-fibre direction. The volume fraction of actuator was varied in the range 0 to 2.5 vol\%. These laminates were actuated electrically and were designed to exhibit macroscopic shape changes during actuation. The work presented in this paper was a preliminary appraisal of the durability issues associated with the use of such hybrid composites. This has shown that actuator fraction plays a significant role in controlling both the shape change obtained from these materials and the hysteresis associated with their forward and reverse actuation. It has also been shown that during cyclic operation hybrid laminates can suffer from instabilities in shape change due to the accumulation of internal damage. However, by careful selection of the maximum actuation strain and actuator fraction it is possible to achieve useable and stable cyclic shape changes.
\end{abstract}

\section{INTRODUCTION}

Imagine an engineering system capable of operating at the very limit of its performance envelope without fear of exceeding its structural limits. Imagine such a structure also having the ability to change shape on demand, diagnose damage and carry out self-repair. These concepts are no longer pipe-dreams and science fact is approaching science fiction with the advent of 'Smart' structures. 'Smart' structures are an emerging technology which will provide the possibility of engineering structures with enhanced functionality for a wide range of applications. Such structures will provide, for example, enhanced vibration control and real-time aeroelastic tailoring of structures, and real-time health-monitoring of safety critical systems. To produce this enhanced performance 'Smart' structures require internal mechanisms through which they can sense their environment, process this sensory data, and respond to external stimuli. In most current Smart Structural Concepts a mechatronic or 'Frankenstein' approach is adopted where separate sensors, signal processing and actuators are 'boltedtogether' to produce a 'Smart' system response. In the majority of these concepts the sensors and actuators are integrated within the host structure itself, and many of the sensors and actuators are familiar from more conventional sensing/actuation applications. Amongst the materials proposed for actuators are Shape-Memory Alloys (SMAs) since they offer the possibility of high strain/stress actuation. 
The literature-base on the integration of SMA actuators into hybrid composite structures is not extensive. However, their use has been investigated for vibration [1], acoustic radiation [1,2], damage [3], buckling [1,2], and shape [1] control. An interesting feature of this work has been a heavy bias towards modelling, with only limited attempts to experimentally verify the calculated results. Previous work has also failed to produce a systematic database on another key issue. This is the durability of these hybrid composites. The present work was therefore undertaken to provide a preliminary appraisal of the durability issues associated with such systems.

\section{EXPERIMENTAL DETAILS}

There are a variety of ways in which SMA hybrid composites can be employed, each with their own specific durability issues. However, one implementation of these materials allows an extremely simple appraisal of their behaviour. This is to employ systems where the actuators develop macroscopic shapechanges of a composite structure. The performance of such 'active' composites can then be easily characterised by their external shape-change, and the stability of this property can be investigated as a function of key experimental variables. This approach was adopted in the present work.

The samples employed in this programme were small glass-fibre reinforced epoxy cantilever beams $(145 \times 20 \mathrm{~mm})$ containing embedded Ni-Ti SMA actuators. The beams were constructed by hand lay-up and vacuum-bagging using Fibredux 913 unidirectional glass fibre pre-preg. The actuators were present in the form of $300 \mu \mathrm{m}$ diameter wires with a transformation temperature $\left(\mathrm{A}_{\mathrm{f}}\right)$ of $60^{\circ} \mathrm{C}$. In the reported work the actuators were orientated parallel to the fibre direction of the unidirectional composite beams. The beams were 6 ply in thickness with the actuators introduced between plies 2 and 3 , and the volume fraction of actuator was varied systematically in the range 0 to $2.5 \mathrm{vol} \%$. A one-way memory strain of $6 \%$ was initially introduced into the actuators by restraining the Ni-Ti wires in a custom-built jig. The composite beams were then built around the strained actuators. This $6 \%$ strain was maintained on the actuators during composite cure which was carried out using a thermal cycle recommended by the pre-preg manufacturer for the vacuum-bag processing of Fibredux 913 . Once the cure cycle was complete the hybrid beams were removed from their fixing jigs and prior to testing the actuators were restrained against the free ends of the beams. The latter was carried out since preliminary tests showed that in unconstrained hybrid beams actuation resulted in significant interfacial decohesion between the actuator and host composite which resulted in severe degradation of the actuation strains.

Since the actuators were off the beams' neutral axes bending was induced during actuation. Such deflections were used to characterise the mechanical responses of the hybrid beams. Measurement of these deflections (to an accuracy of $\pm 30 \mu \mathrm{m}$ ) was by means of a commercial non-contact optical displacement measurement system. This employed a laser and operated by simple triangulation. During the first actuation cycles the deflections of the beams resulted from the $6 \%$ one-way memory strain introduced during laminate manufacture. However, during subsequent cycles, actuation resulted from re-setting of the memory strain during the cooling stage of the previous cycle. This occurred since the actuators operated against the elastic stiffness of the host laminate which imposed a bias force on the actuators throughout their operating cycle (and most particularly during the forward parent to martensite transformation).

Laminate shape changes were activated electrically by means of resistive heating, with the actuators connected in series to ensure that each volume element was subjected to the same heating current. During deflection testing the heating was increased over a period of approximately 2 seconds and was then allowed to stabilise for one minute prior to measurements being taken. Heating was therefore increased incrementally up to the maximum value for each cycle. The mechanical performance of the SMA hybrid beams were initially characterised by their deflection/ current response. However, since the true drive variable for the actuators was temperature, experiments were also conducted to crosscorrelate drive currents to actuator temperature rises. This was done by the use of fine thermocouples 
embedded within the host composites close to the actuators. Optical and electron metallography was also employed to investigate damage accumulation within the host composites during cyclic actuation of the laminates.

\section{RESULTS AND DISCUSSION}

Figure 1 shows the microstructure of a typical hybrid laminate with the $300 \mu \mathrm{m}$ diameter SMA actuator embedded within the glass-fibres of the host composite. With the Fibredux 913 pre-preg system there was a limit to the maximum temperature to which the laminates could be driven. This was an actuator temperature of approximately $150^{\circ} \mathrm{C}$. If actuators were driven beyond this level there was visible darkening of the host composite around the actuators, and delamination was observed through the transluscent resin. This restricted all measurements to maximum actuator temperatures below this level.

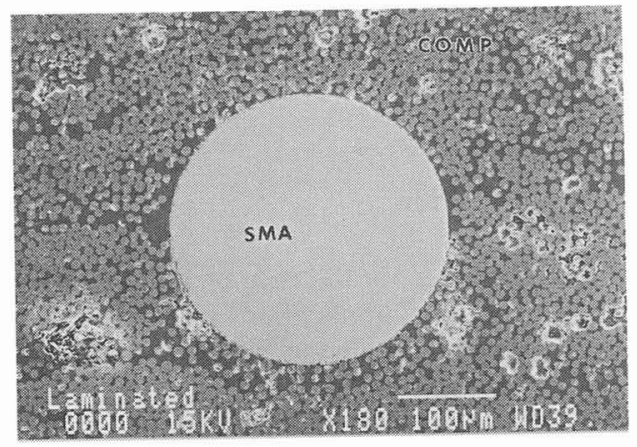

Figure 1 - Typical Hybrid Laminate

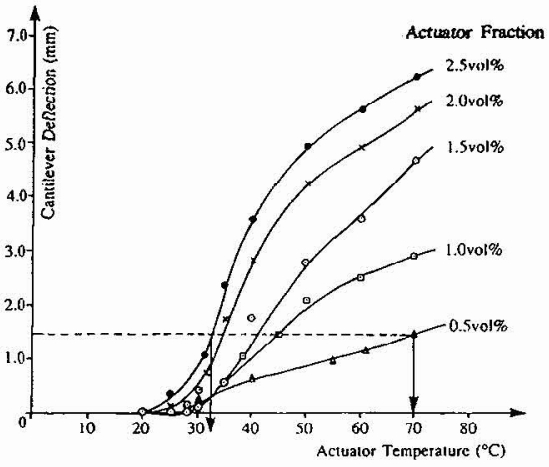

Figure 2 - Effect of Actuator Fraction on Deflection Output

Figure 2 shows the effect of actuator fraction on the deflection characteristics of the hybrid beams (actuator strain and beam stiffness being approximately constant). It can be clearly seen that as the volume fraction of actuator increased the maximum deflections of the cantilever beams also increased. Such observations are not unexpected since in the higher actuator fraction laminates there was greater recovery force available from the multiple actuators for elastic deformation of the host composite. Thus it would be expected that higher actuator fraction beams would generate larger cantilever deflections. However, figure 2 also has implications for the control of such hybrid beams. This data shows that it is possible to develop identical macroscopic shape-changes using two different strategies. One approach would be to 'over-actuate' (ie heat to higher temperatures) lower actuator fraction composites. Alternatively, the same shape-change could be achieved by partially actuating (to lower temperatures) a higher fraction material. These two approaches clearly have different effects on laminate durability (particularly on issues relating to heat input) and it will be shown later that they also have different effects on the longer-term cyclic stabilities of the laminates.

Figure 3 shows both heating and cooling stages of actuation cycles for three of the hybrid beams. This shows the strong dependence of hysteresis on the volume fraction of actuator. This is interesting since similar changes in the form of deflection/ temperature curves are often observed in more conventional SMA applications where actuators are employed against varying levels of external bias force [4,5]. In such conventional applications, operating actuators against increasing 'spring-rates' (where the bias force increases as a function of actuation strain) results in decreased strain outputs, lower temperature sensitivity of the strain output, and smaller forward/reverse hysteresis. 


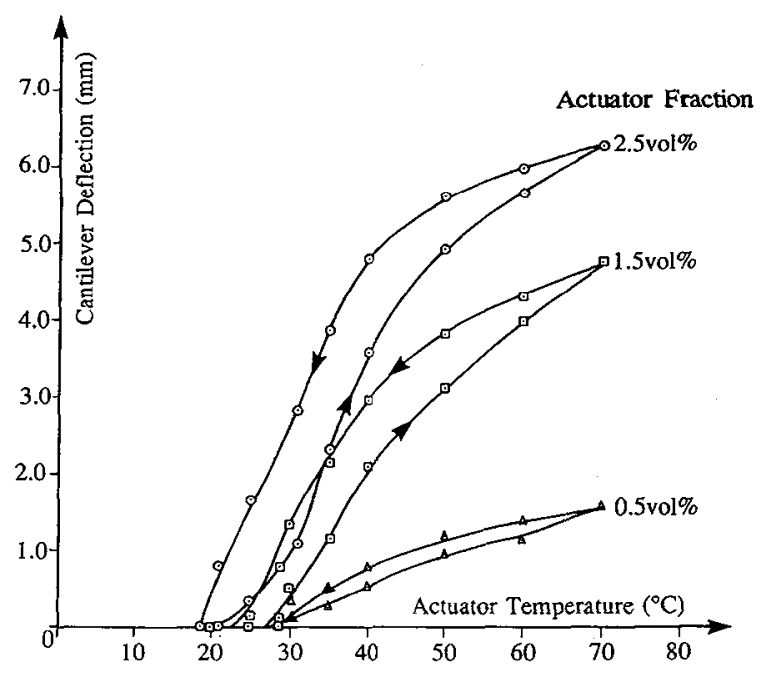

Figure 3 - Hysteresis Associated with Actuation

Phenomenologically the observations in figure 3 appear similar. However, in the case of these hybrid laminates the origin of this behaviour is not as simple as that in conventional applications since the biasrates (the stiffness of the host composites) were approximately constant, but the actuator fractions varied. Currently it is not possible to provide a categorical explanation for the wider hysteresis at higher actuator fractions, however, it is believed that it is associated with both the restoring force developed in the hybrid beams during actuation, and the effect of this force on the forward (parent to martensite) transformation within the actuator during cooling.

The increase in shape-change with increasing actuator fraction has been discussed above. This clearly arose since the presence of multiple actuators allowed a large laminate deflection to be developed without individual actuators being subjected to high bias stresses. Thus although higher bias forces were produced in the higher actuator fraction laminates (due to the larger deflections of beams of constant stiffness), the increased area fraction of actuator resulted in lower effective stresses on the actuators. This clearly resulted in the higher cantilever deflections and the higher temperature sensitivity of the deflection output (as seen in both figure 2 and 3). Thus the actuators in higher actuator fraction materials behaved as if they were subjected to lower effective bias-rates. It is now widely reported [6] that such conditions also alter the hysteresis width, with wider hysteresis observed at lower bias rates. This was therefore the most likely origin of the differences in hysteresis shown in figure 3.

The behaviour shown in figures 2 and 3 are the first cycle responses of the beams. However, when considering the durability of these materials it is perhaps more important to consider the longer-term stability of such behaviour. Figure 4 shows data of this type for the 1 vol\% material investigated in this work. The laminates employed for these measurements were cycled to a maximum actuator temperature of $150^{\circ} \mathrm{C}$. It is clear that deflection data from these measurements differed slightly from that shown in figure 2. It is believed that this is due to laminate to laminate variations, compounded with a small systematic error in the conversion of drive current to actuator temperature rise. However, this data does show that there was a systematic instability in response, with changes in deflection output over the first three actuation cycles. After these initial cycles a more stable behaviour developed. 


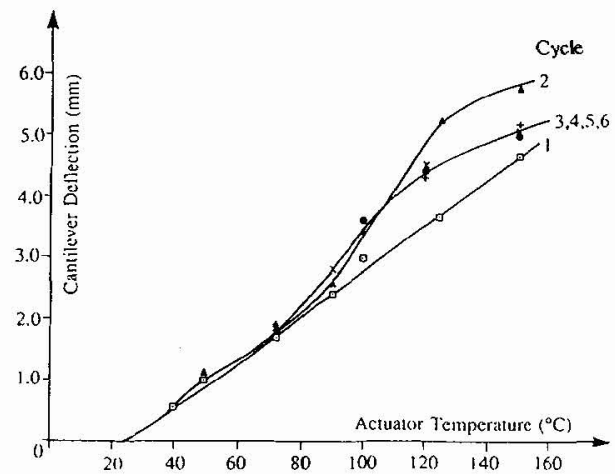

Figure 4 - Cyclic Data for 1 vol\% Actuator

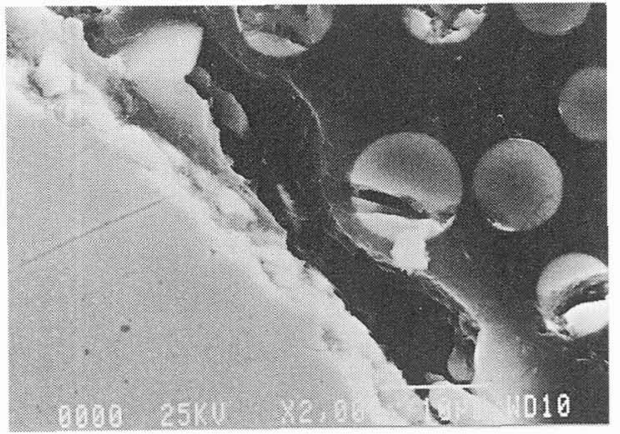

Figure 5 - Actuator/ Host Interfacial Failure

The origin of such instabilities in deflection output were investigated in terms of damage accumulation within the cycled composites. Microscopy showed that during the first three actuation cycles damage was initiated within the beams. This internal damage was in the form of interfacial breakdown between the actuators and host composite (figure 5).

The actuator/composite interface gradually degraded with each actuation cycle until cycle 3 where gross delamination was present. This is interesting since figure 4 shows that this cycle coincided with the development of a reproducible deflection response. This suggests that during the first three cycles interfacial contact gradually decreased until the actuators were completely delaminated within the host composite and acted on the beams only through the mechanical constraints at the free ends. Following cycle 3 no further delamination was possible and there were then no further changes in the deflection responses of the beams. It is also interesting to note that during this delamination the deflection ouput from the beams actually increased.

It is possible to investigate further this instability in deflection response by replotting the data in figure 4. This is shown in figure 6 which presents the cycle to cycle stability of the deflection outputs at a number of different temperatures.

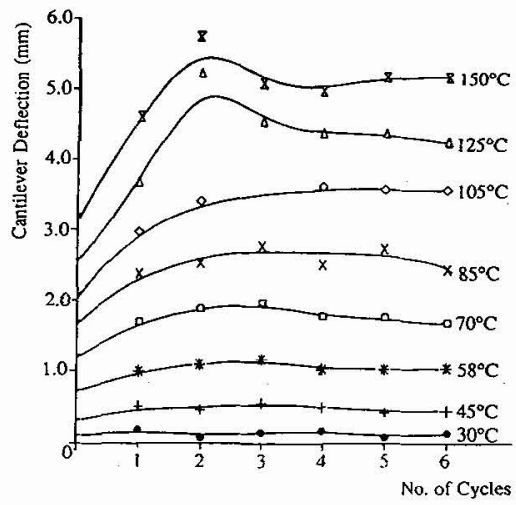

Figure 6 - Cyclic Stability of Deflection Outputs at Temperatures between 30 and $150^{\circ} \mathrm{C}$

This data essentially highlights the regions of the original deflection/temperature curves (figure 4) with the greatest cycle to cycle instability. It is clear from figure 6 that although there was some cycle to 
cycle drift in the deflection outputs at all temperatures, the greatest instability was at higher values. This suggests that if the maximum cycling temperature is decreased, improved cyclic stability should result. This was confirmed by cycling beams to a lower maximum temperature. Typical data is shown in figures 7(a) and (b).
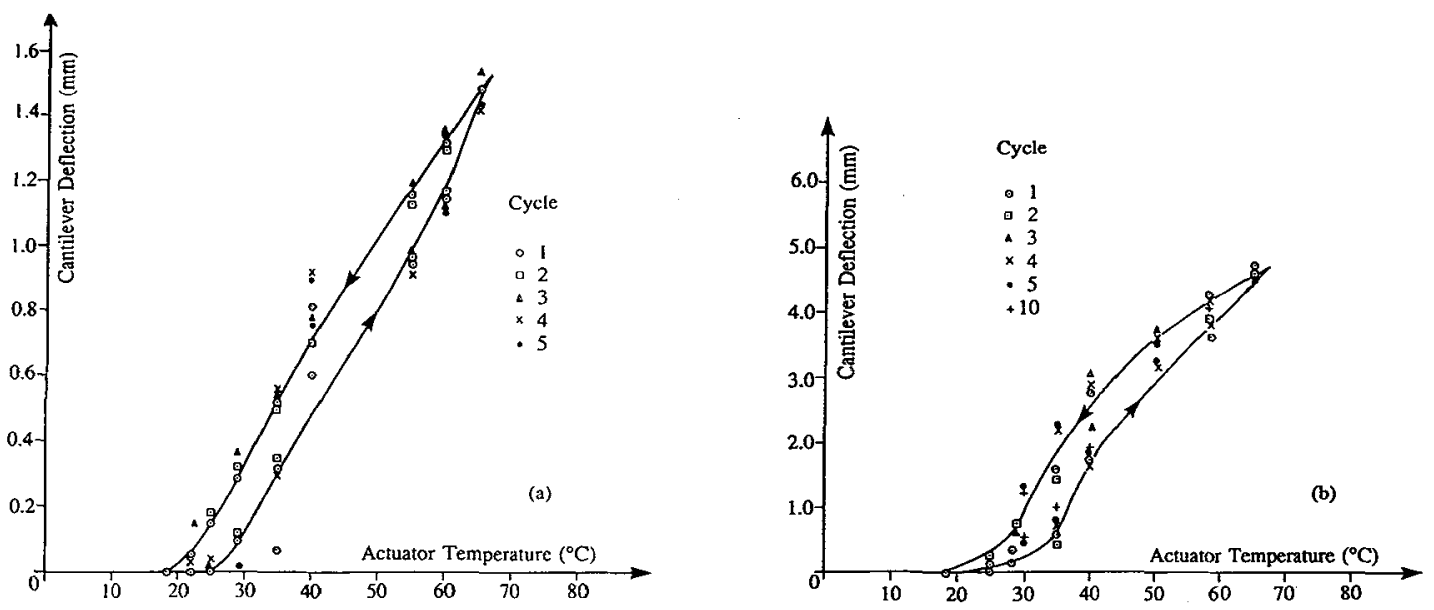

Figure 7 - Cyclic Data for (a) 0.5 , and (b) 1.5 vol\% SMA Hybrid Composites (maximum actuator temperature $65^{\circ} \mathrm{C}$ )

Cycling to $65^{\circ} \mathrm{C}$ resulted in reproducible behaviour with respect to both the forward and reverse actuation pathways. The deflection outputs were smaller than those obtained by cycling to higher temperatures, however, the absolute value could be maximised to some extent by selection of an appropriate actuator fraction.

\section{CONCLUSIONS}

The results presented in this paper begin to address some of the durability issues associated with the use of SMA hybrid composite materials. It has been shown that as expected actuator fraction plays a significant role in controlling both the deflection response of these composites and the hysteresis associated with their forward and reverse actuation pathways. It has also been shown that during cyclic operation such hybrid composites can suffer from instabilities in deflection response. This appears to be associated with the accumulation of internal damage within the composites due to cycling close to the maximum temperature capability of these materials. However, it has also been shown that by careful selection of the maximum temperature rise during actuation and the volume fraction of actuator, it is possible to achieve useable deflection outputs which are stable over many actuation cycles.

\section{REFERENCES}

[1] Liang C. Jia J. C Rogers., Proc 30 ${ }^{\text {th }}$ AIAA/ASME/ASCE/AHS/ASC Structures, Structural Dynamics and Materials Conf. (AIAA, 1989) pp 1504 - 1513

[2] Thompson D.H. Griffin O.H., Proc Conf Recent Advances in Adaptive and Sensory Materials and their Applications, Blacksburg, 1992 (Technomic, 1992) pp $377-384$

[3] Rogers C.A, Liang C. Li S., Proc AIAA 32 ${ }^{\text {nd }}$ Structures, Structural Dynamics and Materials, Baltimore, 1991

[4] Friend C.M., Scripta Met 20 (1986) 995

[5] Melton K.N. Mercier O., Scripta Met 12 (1978) 5

[6] Michael A.D. Hart W.B., Met \& Mat Tech 6 (1980) 434 